\title{
Gromov-Witten Invariants in Algebraic Geometry
}

\author{
K. Behrend
}

January 13, 1996

\begin{abstract}
Gromov-Witten invariants for arbitrary projective varieties and arbitrary genus are constructed using the techniques from $[\mathrm{K}$. Behrend, B. Fantechi. The Intrinsic Normal Cone.]
\end{abstract}

\section{Introduction}

In [2] the problem of constructing the Gromov-Witten invariants of a smooth projective variety $V$ was reduced to defining a 'virtual fundamental class'

$$
\left[\bar{M}_{g, n}(V, \beta)\right]^{\mathrm{virt}} \in A_{(1-g)(\operatorname{dim} V-3)-\beta\left(\omega_{V}\right)+n}\left(\bar{M}_{g, n}(V, \beta)\right)
$$

in the Chow group of the algebraic stack

$$
\bar{M}_{g, n}(V, \beta)
$$

of stable maps of class $\beta \in H_{2}(V)$ from an $n$-marked prestable curve of genus $g$ to $V$.

If $g=0$ and $V$ is convex (i.e. $H^{1}\left(\mathbb{P}^{1}, f^{*} T_{V}\right)=0$, for all $f: \mathbb{P}^{1} \rightarrow V$ ), then $\bar{M}_{0, n}(V, \beta)$ is smooth of the expected dimension $\operatorname{dim} V-3-\beta\left(\omega_{V}\right)+n$ and the usual fundamental class

$$
\left[\bar{M}_{g, n}(V, \beta)\right]
$$

will work. This was proved in [2].

In this paper we treat the general case using the construction from [1]. Recall from [ibid.] that virtual fundamental classes are constructed using an obstruction theory, and the intrinsic normal cone. The obstruction theory serves to give rise to a vector bundle stack $\mathfrak{E}$, into which the intrinsic normal cone $\mathfrak{C}$ can be embedded as a closed subcone stack. The virtual fundamental class is then obtained by intersecting $\mathfrak{C}$ with the zero section of $\mathfrak{E}$. 
In our context, this process works as follows. Let $\mathfrak{M}_{g, n}$ be the algebraic stack of $n$-marked prestable curves of genus $g$. This is an algebraic stack, not of Deligne-Mumford (or even finite) type, but smooth of dimension $3(g-1)+n$. There is a canonical morphism

$$
\bar{M}_{g, n}(V, \beta) \rightarrow \mathfrak{M}_{g, n}
$$

given by forgetting the map, retaining the curve (but not stabilizing). Then $\bar{M}_{g, n}(V, \beta) \rightarrow \mathfrak{M}_{g, n}$ is an open substack of a stack of morphisms, and as such has a relative obstruction theory, which in this case is $\left(R \pi_{*} f^{*} T_{V}\right)^{\vee}$, where $\pi: C \rightarrow \bar{M}_{g, n}(V, \beta)$ is the universal curve and $f: C \rightarrow V$ is the universal stable map. Saying that $\left(R \pi_{*} f^{*} T_{V}\right)^{\vee}$ is a relative obstruction theory means that there is a homomorphism

$$
\phi:\left(R \pi_{*} f^{*} T_{V}\right)^{\vee} \longrightarrow L_{\bar{M}_{g, n}(V, \beta) / \mathfrak{M}_{g, n}},
$$

(where $L^{\bullet}$ is the cotangent complex) such that $h^{0}(\phi)$ is an isomorphism and $h^{-1}(\phi)$ is surjective.

The homomorphism $\phi$ induces a closed immersion

$$
\phi^{\vee}: \mathfrak{N}_{\bar{M}_{g, n}(V, \beta) / \mathfrak{M}_{g, n}} \longrightarrow h^{1} / h^{0}\left(R \pi_{*} f^{*} T_{V}\right)
$$

of abelian cone stacks (see [1]) over $\bar{M}_{g, n}(V, \beta)$, where $\mathfrak{N}$ is the relative intrinsic normal sheaf. The relative intrinsic normal cone $\mathfrak{C}_{\bar{M}_{g, n}(V, \beta) / \mathfrak{M}_{g, n}}$ is a closed subcone stack of $\mathfrak{N}_{\bar{M}_{g, n}(V, \beta) / \mathfrak{M}_{g, n}}$, and so we get a closed immersion of cone stacks

$$
\mathfrak{C}_{\bar{M}_{g, n}(V, \beta) / \mathfrak{M}_{g, n}} \longrightarrow h^{1} / h^{0}\left(R \pi_{*} f^{*} T_{V}\right) .
$$

Now since $R \pi_{*} f^{*} T_{V}$ has global resolutions (see Proposition 5), we may intersect $\mathfrak{C}_{\bar{M}_{g, n}(V, \beta) / \mathfrak{M}_{g, n}}$ with the zero section of the vector bundle stack $h^{1} / h^{0}\left(R \pi_{*} f^{*} T_{V}\right)$ to get the virtual fundamental class $\left[\bar{M}_{g, n}(V, \beta)\right]^{\mathrm{virt}}$.

The fundamental axioms (see [5]) Gromov-Witten invariants need to satisfy to deserve their name are reduced in [2] to five basic compatibilities between the virtual fundamental classes. These follow from the basic properties proved in [1]. The dimension axiom, for example, follows from the basic fact that the intrinsic normal cone always has dimension zero.

We also show that if $V=G / P$, for a reductive group $G$ and a parabolic subgroup $P$, there is an alternative construction of the virtual fundamental classes avoiding the intrinsic normal cone. We construct a cone $C$ in the vector bundle $R^{1} \pi_{*} \mathcal{O} \otimes \mathfrak{g}$ on $\bar{M}_{g, n}(V, \beta)$, which may then be intersected with 
the zero section of $R^{1} \pi_{*} \mathcal{O} \otimes \mathfrak{g}$ to obtain the virtual fundamental class. This cone $C$ is constructed as the normal cone of an embedding of $\bar{M}_{g, n}(V, \beta)$ into a certain stack of principal $P$-bundles (which is smooth, but not of Deligne-Mumford type).

A construction of Gromov-Witten invariants using a cone inside a vector bundle has also been announced by J. Li and G. Tian. Their methods differ from ours in that they use analytic methods, including the Kuranishi map.

Most of this work was done during a stay at the Max-Planck-Institut für Mathematik in Bonn, and I would like to take this opportunity to acknowledge the hospitality and the wonderful atmosphere at the MPI. I would also like to thank Professors G. Harder and Yu. Manin for fruitful discussions about the constructions in this paper.

\section{Preliminaries on Prestable Curves}

Let $k$ be a field. We shall work over the category of locally noetherian $k$-schemes (with the fppf-topology). For a modular graph $\tau$ (see [2], Definition 1.5) let $\mathfrak{M}(\tau)$ denote the $k$-stack of $\tau$-marked prestable curves (which are defined in [2], Definition 2.6).

Lemma 1 The algebraic $k$-stack $\bar{M}(\tau)$ of stable $\tau$-marked curves is an open substack of $\mathfrak{M}(\tau)$.

Proof. Let $\mathcal{C}_{v} \rightarrow \mathfrak{M}(\tau)$ be the universal curve corresponding to the vertex $v \in V_{\tau}$. Let $\widetilde{\mathcal{C}_{v}}$ be the stabilization. Then $\bar{M}(\tau)$ is the substack of $\mathfrak{M}(\tau)$ over which all $p_{v}: \mathcal{C}_{v} \rightarrow \widetilde{\mathcal{C}}_{v}$ are isomorphisms. This is open because the $\mathcal{C}_{v}$ are proper over $\mathfrak{M}(\tau)$.

Now consider a modular graph $\tau^{\prime}$ obtained from $\tau$ by adding some tails. We get an induced morphism of $k$-stacks $\mathfrak{M}\left(\tau^{\prime}\right) \rightarrow \mathfrak{M}(\tau)$ which simply forgets the markings corresponding to the tails $S_{\tau^{\prime}}-S_{\tau}$. If $S_{\tau^{\prime}}-S_{\tau}$ has cardinality 1, then $\mathfrak{M}\left(\tau^{\prime}\right) \rightarrow \mathfrak{M}(\tau)$ is a smooth curve, hence representable and smooth of relative dimension 1 . So by induction, $\mathfrak{M}\left(\tau^{\prime}\right) \rightarrow \mathfrak{M}(\tau)$ is representable and smooth of relative dimension \#( $\left.S_{\tau^{\prime}}-S_{\tau}\right)$. By Lemma 11 the same is true for $\bar{M}\left(\tau^{\prime}\right) \rightarrow \mathfrak{M}(\tau)$.

Proposition 2 The stack $\mathfrak{M}(\tau)$ is a smooth algebraic $k$-stack of dimension

$$
\operatorname{dim}(\tau)=\# S_{\tau}-\# E_{\tau}-3 \chi(\tau)
$$


Proof. For the definition of $\operatorname{dim}(\tau)$ and $\chi(\tau)$ see [2], Definitions 6.1 and 6.2.

Note that for every point of $\mathfrak{M}(\tau)$ there exists a $\tau^{\prime}$ as above such that the induced morphism $\bar{M}\left(\tau^{\prime}\right) \rightarrow \mathfrak{M}(\tau)$ contains this given point in its image. Thus $\coprod_{\tau^{\prime}} \bar{M}\left(\tau^{\prime}\right)$ is a presentation of $\mathfrak{M}(\tau)$ showing that $\mathfrak{M}(\tau)$ is algebraic.

Now let $\tau^{s}$ be the stabilization of $\tau$. Stabilization defines a morphism of algebraic $k$-stacks

$$
s: \mathfrak{M}(\tau) \longrightarrow \bar{M}\left(\tau^{s}\right) .
$$

If $\tau^{\prime}$ is obtained as above by adjoining tails to $\tau$ such that $\tau^{\prime}$ is stable, we have a commutative diagram

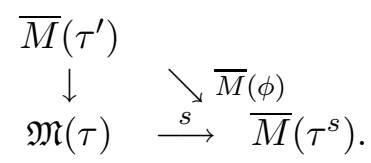

Here $\phi: \tau^{\prime} \rightarrow \tau^{s}$ is the canonical morphism of stable modular graphs. In fact, one may define $s$ locally by using such diagrams.

Proposition 3 The morphism $s: \mathfrak{M}(\tau) \rightarrow \bar{M}\left(\tau^{s}\right)$ is flat.

Proof. This follows by descent since the morphism $\bar{M}(\phi)$ for various $\phi$ : $\tau^{\prime} \rightarrow \tau^{s}$ as above are flat.

\section{The Virtual Fundamental Classes}

Over $\mathfrak{M}(\tau)$ there is a family $\left(\mathcal{C}_{v}\right)_{v \in V_{\tau}}$ of universal curves, with sections $x_{i}$ : $\mathfrak{M}(\tau) \rightarrow \mathcal{C}_{\partial_{\tau}(i)}$. Let $\mathcal{C}(\tau) \rightarrow \mathfrak{M}(\tau)$ be the curve obtained from $\coprod_{v \in V_{\tau}} \mathcal{C}_{v}$ by identifying $x_{i}$ and $x_{j}$, for every edge $\{i, j\} \in E_{\tau}$. The curve $\mathcal{C}(\tau)$ has markings $x_{i}: \mathfrak{M}(\tau) \rightarrow \mathcal{C}(\tau)$, for each $i \in S_{\tau}$. In fact, $\mathcal{C}(\tau)$ is a $\widetilde{\tau}$-marked prestable curve, where $\widetilde{\tau}$ is the graph obtained from $\tau$ by contracting all edges of $\tau$. Let us denote the structure morphism by

$$
\pi: \mathcal{C}(\tau) \longrightarrow \mathfrak{M}(\tau)
$$

We shall also denote any base change of $\pi$ by $\pi$.

Now let $V$ be a smooth projective $k$-variety, $(\tau, \beta)$ a stable $V$-graph and let $\operatorname{Mor}_{\mathfrak{M}(\tau)}(\tau, V)$ be the $\mathfrak{M}(\tau)$-space of morphisms from $\mathcal{C}(\tau)$ to $V$. Denote the universal morphism by

$$
f: \mathcal{C}(\tau) \times \operatorname{Mor}_{\mathfrak{M}(\tau)}(\tau, V) \longrightarrow V \text {. }
$$


By [3] the stack $\operatorname{Mor}_{\mathfrak{M}(\tau)}(\tau, V)$ is an algebraic $k$-stack and the structure morphism

$$
\operatorname{Mor}_{\mathfrak{M}(\tau)}(\tau, V) \longrightarrow \mathfrak{M}(\tau)
$$

is representable.

Proposition 4 The proper Deligne-Mumford stack $\bar{M}(V, \tau, \beta)$ of stable maps is an open substack of $\operatorname{Mor}_{\mathfrak{M}(\tau)}(\tau, V)$.

Proof. The set of points where stabilization is an isomorphism is open.

To define the virtual fundamental class on $\bar{M}(V, \tau, \beta)$ we consider the morphism $\bar{M}(V, \tau, \beta) \rightarrow \mathfrak{M}(\tau)$ and denote the relative intrinsic normal cone (see [1]) by

$$
\mathfrak{C}(V, \tau, \beta)=\mathfrak{C}_{\bar{M}(V, \tau, \beta) / \mathfrak{M}(\tau)}
$$

The intrinsic normal sheaf [ibid.] of $\bar{M}(V, \tau, \beta)$ over $\mathfrak{M}(\tau)$ we shall denote by $\mathfrak{N}(V, \tau, \beta)$.

By the relative version of [1] Proposition 6.2 we have a perfect relative obstruction theory [ibid.]

$$
\pi_{*}\left(e^{\vee}\right)^{\vee}: R \pi_{*}\left(f^{*} T_{V}\right)^{\vee} \longrightarrow L_{\dot{M o r}_{\mathfrak{M}(\tau)}(\tau, V) / \mathfrak{M}(\tau)} .
$$

Restricting to the open substack $\bar{M}(V, \tau, \beta)$ we get a perfect relative obstruction theory

$$
\pi_{*}\left(e^{\vee}\right)^{\vee}: R \pi_{*}\left(f^{*} T_{V}\right)^{\vee} \longrightarrow L_{\bar{M}(V, \tau, \beta) / \mathfrak{M}(\tau)}^{\bullet}
$$

which we shall also denote by $E^{\bullet}(V, \tau, \beta)$. Thus $\mathfrak{C}(V, \tau, \beta)$ is embedded as a closed subcone stack in the vector bundle stack

$$
\mathfrak{E}(V, \tau, \beta)=h^{1} / h^{0}\left(R \pi_{*} f^{*} T_{V}\right) .
$$

Note that the relative virtual dimension of $\bar{M}(V, \tau, \beta)$ over $\mathfrak{M}(\tau)$ with respect to the obstruction theory $R \pi_{*}\left(f^{*} T_{V}\right)^{\vee}$ is equal to

$$
\begin{aligned}
\operatorname{rk} R \pi_{*}\left(f^{*} T_{V}\right)^{\vee} & =\chi\left(f^{*} T_{V}\right) \\
& =\operatorname{deg} f^{*} T_{V}+\operatorname{dim} V \cdot \chi(\mathcal{C}(\tau)) \\
& =\chi(\tau) \operatorname{dim} V-\beta(\tau)\left(\omega_{V}\right) .
\end{aligned}
$$

Essential is the following result. 
Proposition 5 Let $(C, x, f)$ be a stable map over $T$ to $V$, where $T$ is a finite type algebraic $k$-stack. Let $E$ be a vector bundle on $C$. Then $R \pi_{*} E$ has global resolutions, where $\pi: C \rightarrow T$ is the structure map.

Proof. Let $M$ be an ample invertible sheaf on $V$ and let

$$
L=\omega_{C / T}\left(x_{1}+\ldots+x_{n}\right) \otimes f^{*} M^{\otimes 3} .
$$

By Proposition 3.9 of [2] the sheaf $L$ is ample on the fibers of $\pi$. So for sufficiently large $N$ we have that

1. $\pi^{*} \pi_{*}\left(E \otimes L^{\otimes N}\right) \rightarrow E \otimes L^{\otimes N}$ is surjective,

2. $R^{1} \pi_{*}\left(E \otimes L^{\otimes N}\right)=0$,

3. for all $t \in T$ we have that $H^{0}\left(C_{t}, L_{t}^{\otimes-N}\right)=0$.

Let

$$
F=\pi^{*} \pi_{*}\left(E \otimes L^{\otimes N}\right) \otimes L^{\otimes-N}
$$

and let $H$ be the kernel of the map $F \rightarrow E$. Thus we have a short exact sequence

$$
0 \longrightarrow H \longrightarrow F \longrightarrow E \longrightarrow 0
$$

of vector bundles on $C$. Note that for every $t \in T$ we have

$$
\begin{aligned}
H^{0}\left(C_{t}, F\right) & =H^{0}\left(C_{t}, \pi_{*}\left(E \otimes L^{\otimes N}\right)_{t} \otimes L_{t}^{\otimes-N}\right) \\
& =H^{0}\left(C_{t}, L_{t}^{\otimes-N}\right) \otimes \pi_{*}\left(E \otimes L^{\otimes N}\right)_{t} \\
& =0
\end{aligned}
$$

and hence $H^{0}\left(C_{t}, H\right)=0$, also. Therefore, $\pi_{*} H$ and $\pi_{*} F$ are zero and $R^{1} \pi_{*} H$ and $R^{1} \pi_{*} F$ are locally free. This implies that

$$
R \pi_{*} E \cong\left[R^{1} \pi_{*} H \rightarrow R^{1} \pi_{*} F\right]
$$

As shown in [1], by Proposition 5 the obstruction theory $R \pi_{*}\left(f^{*} T_{V}\right)^{\vee}$ gives rise to a virtual fundamental class

$$
\left[\bar{M}(V, \tau, \beta), R \pi_{*}\left(f^{*} T_{V}\right)^{\vee}\right] \in A_{\operatorname{dim}(V, \tau, \beta)}(\bar{M}(V, \tau, \beta)),
$$


since

$$
\begin{aligned}
\operatorname{dim} & \mathfrak{M}(\tau)+\operatorname{rk} R \pi_{*}\left(f^{*} T_{V}\right)^{\vee} \\
\quad= & \chi(\tau)(\operatorname{dim} V-3)-\beta(\tau)\left(\omega_{V}\right)+\# S_{\tau}-\# E_{\tau} \\
\quad= & \operatorname{dim}(V, \tau, \beta) .
\end{aligned}
$$

(See Definition 6.2 in [2] for the definition of $\operatorname{dim}(V, \tau, \beta)$.)

Theorem 6 The system of virtual fundamental classes

$$
J(V, \tau, \beta)=\left[\bar{M}(V, \tau, \beta), R \pi_{*}\left(f^{*} T_{V}\right)^{\vee}\right]
$$

is an orientation of $\bar{M}$ over $\mathfrak{G}_{s}(V)$. If $V$ is convex, on the tree level subcategory $\mathfrak{T}_{s}(V)$, we get back the orientation of [7], Theorem 7.5.

Proof. If $V$ is convex and $\tau$ a forest, then $R^{1} \pi_{*}\left(f^{*} T_{V}\right)=0$, so that the virtual fundamental class is the usual fundamental class by [1] Proposition 7.3. Thus the virtual fundamental class agrees with the orientation of [2], Theorem 7.5. To check that $J$ is an orientation, we need to check the five axioms listed in [2], Definition 7.1. This shall be done in the next Section.

Remark As shown in [2], we get an associated system of Gromov-Witten classes for $V$.

\section{Checking the Axioms}

\section{AxIOM I. Mapping to a point}

Let $\tau$ be a stable $V$-graph of class zero such that $|\tau|$ is non-empty and connected. As noted in [2] Section 7 we have

$$
\bar{M}(V, \tau, 0)=V \times \bar{M}(\tau)
$$

which is obviously smooth over $\mathfrak{M}(\tau)$. In fact, the morphism $\bar{M}(V, \tau, 0) \rightarrow$ $\mathfrak{M}(\tau)$ is just the composition

$$
V \times \bar{M}(\tau) \longrightarrow \bar{M}(\tau) \longrightarrow \mathfrak{M}(\tau)
$$

of projection followed by inclusion. If $\widetilde{\pi}: \mathcal{C}(\tau) \rightarrow \bar{M} \tau$ is the universal curve over $\bar{M}(\tau)$, then $\mathcal{C}(V, \tau, 0)=V \times \mathcal{C}(\tau)$ and $\pi: \mathcal{C}(V, \tau, 0) \rightarrow \bar{M}(V, \tau, 0)$ is identified with id $\times \widetilde{\pi}: V \times \mathcal{C}(\tau) \rightarrow V \times \bar{M}(\tau)$. Hence

$$
\begin{aligned}
R^{1} \pi_{*} f^{*} T_{V} & =T_{V} \otimes R^{1} \widetilde{\pi}_{*} \mathcal{O}_{\mathcal{C}(\tau)} \\
& =\mathcal{T}^{(1)}
\end{aligned}
$$


is locally free. So by [1] Proposition 7.3 we have

$$
\begin{aligned}
J(V, \tau, 0) & =c_{\mathrm{rk} R^{1} \pi_{*} f^{*} T_{V}}\left(R^{1} \pi_{*} f^{*} T_{V}\right) \cdot[\bar{M}(V, \tau, 0)] \\
& =c_{g(\tau) \operatorname{dim} V}\left(\mathcal{T}^{(1)}\right) \cdot[\bar{M}(V, \tau, 0)]
\end{aligned}
$$

which is Axiom I.

\section{AxIOM II. Products}

Let $(\sigma, \alpha)$ and $(\tau, \beta)$ be stable $V$-graphs and denote the 'product' by $(\sigma \times$ $\tau, \alpha \times \beta)$. Note that

$$
E^{\bullet}(V, \sigma \times \tau, \alpha \times \beta)=E^{\bullet}(V, \sigma, \alpha) \boxplus E^{\bullet}(V, \tau, \beta),
$$

so by [1] Proposition 7.4 we have

$$
\begin{aligned}
J(V, \sigma \times \tau, \alpha \times \beta) & =\left[\bar{M}(V, \sigma \times \tau, \alpha \times \beta), E^{\bullet}(V, \sigma, \alpha) \boxplus E^{\bullet}(V, \tau, \beta)\right] \\
& =\left[\bar{M}(V, \sigma, \alpha), E^{\bullet}(V, \sigma, \alpha)\right] \times\left[\bar{M}(V, \tau, \beta), E^{\bullet}(V, \tau, \beta)\right] \\
& =J(V, \sigma, \alpha) \times J(V, \tau, \beta),
\end{aligned}
$$

which is the product axiom.

\section{AxIOM III. Cutting Edges}

Use notation as in [2], Section 7, modified as necessary to avoid confusion. Let $\beta$ denote the $H_{2}(V)^{+}$-structure on both $\sigma$ and $\tau$. Write $\mathfrak{M}=\mathfrak{M}(\tau)=$ $\mathfrak{M}(\sigma)$. Consider the cartesian diagram

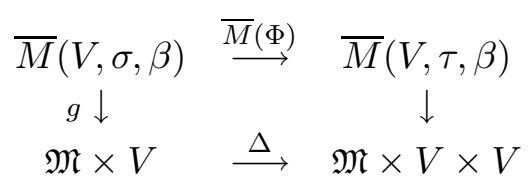

of stacks over $\mathfrak{M}$. Let us show that the obstruction theories $E^{\bullet}(V, \tau, \beta)$ and $E^{\bullet}(V, \sigma, \beta)$ are compatible over $\Delta$ (see «1]).

Over $\bar{M}(V, \sigma, \beta)$ let us consider the following two curves. First the curve $\mathcal{C}=\mathcal{C}(V, \sigma, \beta)$ obtained from the universal curves $\left(C_{v}\right)_{v \in V_{\sigma}}$ by gluing according to the edges of $\sigma$. Secondly, we have the curve $\mathcal{C}^{\prime}$, which we obtain from $\left(C_{v}\right)_{v \in V_{\sigma}}$ by gluing according to the edges of $\tau$. In other words, $\mathcal{C}^{\prime}=\bar{M}(\Phi)^{*} \mathcal{C}(V, \tau, \beta)$. Moreover, $\mathcal{C}$ is obtained from $\mathcal{C}^{\prime}$ by identifying the two sections $x_{1}$ and $x_{2}$ of $\mathcal{C}^{\prime}$, corresponding to the edge $\left\{i_{1}, i_{2}\right\}$ of $\sigma$ which 
is cut by $\Phi$. Thus there is a structure morphism $p: \mathcal{C}^{\prime} \rightarrow \mathcal{C}$ fitting into the commutative diagram

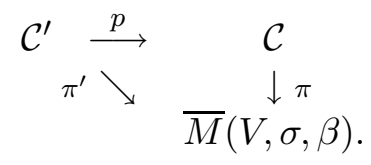

We shall also use the diagram

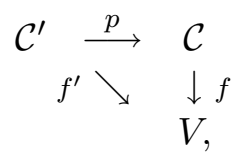

where $f: \mathcal{C} \rightarrow V$ is the universal map. Let $x=p \circ x_{1}=p \circ x_{2}$.

If $E$ is any locally free sheaf on $\mathcal{C}$, then for $i=1,2$ we have the evaluation homomorphism

$$
u_{i}: p^{*} E \longrightarrow x_{i *} x_{i}^{*} p^{*} E=x_{i *} x^{*} E .
$$

Applying $p_{*}$ we get

$$
p_{*}\left(u_{i}\right): p_{*} p^{*} E \longrightarrow x_{*} x^{*} E .
$$

Letting $u=p_{*}\left(u_{2}\right)-p_{*}\left(u_{1}\right)$ we have a short exact sequence

$$
0 \longrightarrow E \longrightarrow p_{*} p^{*} E \stackrel{u}{\longrightarrow} x_{*} x^{*} E \longrightarrow 0
$$

of coherent sheaves on $\mathcal{C}$. Applying $R \pi_{*}$ we get a distinguished triangle

$$
R \pi_{*} E \longrightarrow R \pi_{*}^{\prime} p^{*} E \stackrel{R \pi_{*}(u)}{\longrightarrow} x^{*} E \longrightarrow R \pi_{*} E[1]
$$

in $D\left(\mathcal{O}_{\bar{M}(V, \sigma, \beta)}\right)$. Taking $E=f^{*} T_{V}$ we get the distinguished triangle

$$
R \pi_{*} f^{*} T_{V} \longrightarrow R \pi_{*}^{\prime} f^{*} T_{V} \stackrel{R \pi_{*}(u)}{\longrightarrow} x^{*} f^{*} T_{V} \longrightarrow R \pi_{*} f^{*} T_{V}[1],
$$

or dually,

$$
x^{*} f^{*} \Omega_{V} \stackrel{R \pi_{*}(u)^{\vee}}{\longrightarrow}\left(R \pi_{*}^{\prime} f^{\prime *} T_{V}\right)^{\vee} \longrightarrow\left(R \pi_{*} f^{*} T_{V}\right)^{\vee} \longrightarrow x^{*} f^{*} \Omega_{V}[1] .
$$

Note that we have $E^{\bullet}(V, \sigma, \beta)=\left(R \pi_{*} f^{*} T_{V}\right)^{\vee}$ and $\bar{M}(\Phi)^{*}\left(E^{\bullet}(V, \tau, \beta)\right)=$ $\left(R \pi_{*}^{\prime} f^{\prime *} T_{V}\right)^{\vee}$. Moreover, $L_{\dot{\Delta}}^{\bullet}=\Omega_{V}[1] \mid \mathfrak{M} \times V$, so that $g^{*} L_{\Delta}=x^{*} f^{*} \Omega_{V}[1]$, since $f \circ x=p_{V} \circ g$. So (1) gives the distinguished triangle

$$
g^{*} L_{\Delta}[-1] \stackrel{R \pi_{*}(u)^{\vee}}{\longrightarrow} \bar{M}(\Phi)^{*} E^{\bullet}(V, \tau, \beta) \longrightarrow E^{\bullet}(V, \sigma, \beta) \longrightarrow g^{*} L_{\Delta},
$$


which we may shuffle around to give

$$
\bar{M}(\Phi)^{*} E^{\bullet}(V, \tau, \beta) \longrightarrow E^{\bullet}(V, \sigma, \beta) \longrightarrow g^{*} L_{\Delta} \stackrel{R \pi_{*}(-u)^{\vee}}{\longrightarrow} \bar{M}(\Phi)^{*} E^{\bullet}(V, \tau, \beta)[1] .
$$

Now we have the obstruction morphisms $E^{\bullet}(V, \tau, \beta) \rightarrow L \dot{\bar{M}}_{(V, \tau, \beta) / \mathfrak{M}}$ and $E^{\bullet}(V, \sigma, \beta) \rightarrow L \dot{\bar{M}}_{(V, \sigma, \beta) / \mathfrak{M}}$. Moreover, we have the natural homomorphism $g^{*} L_{\Delta} \rightarrow L_{\bar{M}(\Phi)}^{\bullet}$. These give rise to a homomorphism of distinguished triangles

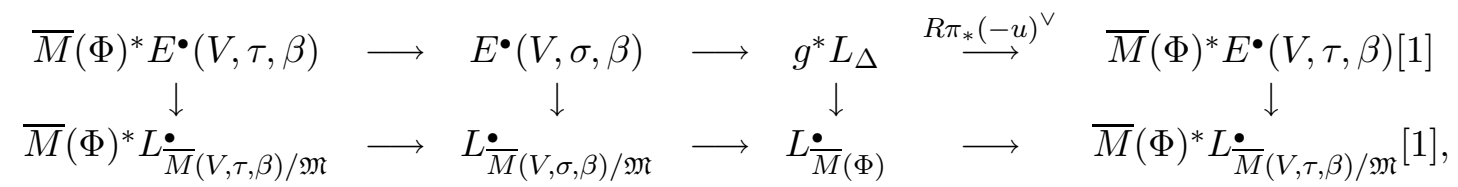

showing that $E^{\bullet}(V, \tau, \beta)$ and $E^{\bullet}(V, \sigma, \beta)$ are compatible over $\Delta$. Hence by [1] Proposition 7.5 we have

$$
\Delta^{!} J(V, \tau, \beta)=J(V, \sigma, \beta)
$$

which is Axiom III.

\section{AxIOM IV. Forgetting Tails}

Let us deal with the incomplete case, leaving the tripod losing cases to the reader. Letting $\mathcal{C} \rightarrow \mathfrak{M}(\tau)$ be the universal curve corresponding to the vertex $w \in V_{\tau}$ (notation from [2], Section 7). We have a cartesian diagram of algebraic $k$-stacks

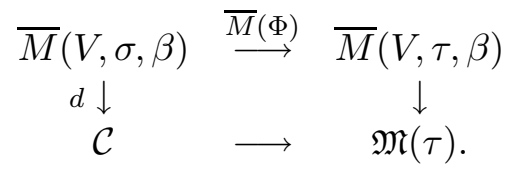

By [1] Proposition 7.2 we have

$$
\bar{M}(\Phi)^{*} J(V, \tau, \beta)=\left[\bar{M}(V, \sigma, \beta), \bar{M}(\Phi)^{*} E^{\bullet}(V, \tau, \beta)\right] .
$$

Here the class on the right hand side is the virtual fundamental class defined by the relative intrinsic normal cone of the morphism $d$ and the relative obstruction theory $\bar{M}(\Phi)^{*} E^{\bullet}(V, \tau, \beta)$. Note that the structure morphism $\bar{M}(V, \sigma, \beta) \rightarrow \mathfrak{M}(\sigma)$ factors through $d: \bar{M}(V, \sigma, \beta) \rightarrow \mathcal{C}$.

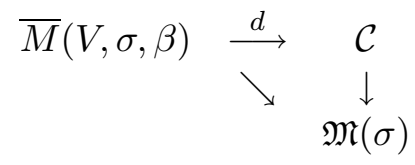


The morphism $d: \bar{M}(V, \sigma, \beta) \rightarrow \mathcal{C}$ associates to the stable map $(C, x, h)$ the pair $\left(\left(C^{\prime}, x^{\prime}\right), y\right)$, where $\left(C^{\prime}, x^{\prime}, h^{\prime}\right)$ is the image of $(C, x, h)$ under $\bar{M}(\Phi)$ and $\left(C^{\prime}, x^{\prime}\right)$ the underlying $\tau$-marked prestable curve. Letting $x_{f}$ be the section of $C_{v}$ corresponding to the flag $f$, we obtain $\left(C^{\prime}, x^{\prime}, h^{\prime}\right)$ by forgetting $x_{f}$ and stabilizing. Moreover, $y$ is the image of the forgotten section $x_{f}$ in $C_{w}^{\prime}$.

The morphism $\mathcal{C} \rightarrow \mathfrak{M}(\sigma)$ associates to the pair $((C, x), y)$, where $(C, x)$ is a $\tau$-marked prestable curve and $y$ a section of $C_{w}$, the $\sigma$-marked prestable curve $(\widetilde{C}, \widetilde{x})$ obtained as follows. For $v^{\prime} \neq v$ we have $\widetilde{C}_{v^{\prime}}=C_{w^{\prime}}$, where $w^{\prime}$ is the vertex of $\tau$ corresponding to $v^{\prime}$. The curve $\left(\widetilde{C}_{v},\left(\widetilde{x}_{j}\right)_{j \in F_{\sigma}(v)}\right)$ is obtained from $\left(\left(C_{w},\left(x_{j}\right)_{j \in F_{\tau}(w)}\right), y\right)$ by 'prestabilizing' (i.e. separating the special points) as in [雨, Definition 2.3.

Lemma 7 The morphism $\mathcal{C} \rightarrow \mathfrak{M}(\sigma)$ is étale.

Proof. We will use the formal criterion for étaleness. Without loss of generality assume that $w$ is the only vertex of $\tau$. So let $((C, x), y)$ be a $\tau$-marked prestable curve with section over the scheme $T, T \rightarrow T^{\prime}$ a square zero extension and $\left(C^{\prime}, x^{\prime}\right)$ a $\sigma$-marked prestable curve over $T^{\prime}$ such that $\left(C^{\prime}, x^{\prime}\right) \mid T$ is the prestabilization of $((C, x), y)$. We may assume that we may choose additional sections $s$ of $C$ over $T$, making $(C, x, s)$ a stable marked curve. Then we extend the sections $s$ to sections $s^{\prime}$ of $C^{\prime}$ over $T^{\prime}$. Taking the stabilization of $\left(C^{\prime}, x^{\prime}, s^{\prime}\right)$ after forgetting the section $x_{f}^{\prime}$ gives an extension of $((C, x), y)$ to $T^{\prime}$ whose prestabilization is $\left(C^{\prime}, x^{\prime}\right)$.

Consider the natural morphism $p: \mathcal{C}(V, \sigma, \beta) \rightarrow \bar{M}(\Phi)^{*} \mathcal{C}(V, \tau)$, which fits into the two commutative diagrams

$$
\begin{array}{ccc}
\mathcal{C}(V, \sigma, \beta) \stackrel{p}{\longrightarrow} & \bar{M}(\Phi)^{*} \mathcal{C}(V, \tau, \beta) \\
& \downarrow & \downarrow \pi^{\prime} \\
& \bar{M}(V, \sigma, \beta)
\end{array}
$$

and

$$
\mathcal{C}(V, \sigma, \beta) \underset{f \searrow}{\stackrel{p}{\longrightarrow}} \bar{M}(\Phi)^{*} \mathcal{C}(V, \tau, \beta)
$$

Whenever $E$ is a locally free sheaf on $\bar{M}(\Phi)^{*} \mathcal{C}(V, \tau, \beta)$ the canonical homomorphism $E \rightarrow p_{*} p^{*} E$ is an isomorphism. Applying this principle to $E=f^{\prime *} T_{V}$ we get an isomorphism

$$
f^{\prime *} T_{V} \longrightarrow p_{*} f^{*} T_{V}
$$


Applying $R \pi_{*}^{\prime}$ to this, gives an isomorphism

$$
R \pi_{*}^{\prime} f^{\prime *} T_{V} \longrightarrow R \pi_{*} f^{*} T_{V} .
$$

Noting that $R \pi_{*}^{\prime} f^{\prime *} T_{V}=\bar{M}(\Phi)^{*} E^{\bullet}(V, \tau, \beta)$ we get an isomorphism

$$
\bar{M}(\Phi)^{*} E^{\bullet}(V, \tau, \beta) \longrightarrow E^{\bullet}(V, \sigma, \beta)
$$

and whence an isomorphism

$$
\mathfrak{E}(V, \sigma, \beta) \longrightarrow \bar{M}(\Phi)^{*} \mathfrak{E}(V, \tau, \beta) .
$$

By [1] Proposition 7.1 there is a natural isomorphism

$$
\mathfrak{C}_{\bar{M}(V, \sigma, \beta) / \mathcal{C}} \longrightarrow \bar{M}(\Phi)^{*} \mathfrak{C}_{\bar{M}(V, \tau, \beta) / \mathfrak{M}(\tau)} .
$$

By Lemma 0 we have a canonical isomorphism

$$
\mathfrak{C}_{\bar{M}(V, \sigma, \beta) / \mathcal{C}} \longrightarrow \mathfrak{C}_{\bar{M}(V, \sigma, \beta) / \mathfrak{M}(\sigma)}
$$

such that the diagram

$$
\begin{array}{rlrl}
\mathfrak{C}_{\bar{M}(V, \sigma, \beta) / \mathfrak{M}(\sigma)} & \stackrel{\sim}{\mathfrak{c}} & \mathfrak{C}_{\bar{M}(V, \sigma, \beta) / \mathcal{C}} \\
\cap & \stackrel{\cap}{\longrightarrow} \bar{M}(\Phi)^{*} \mathfrak{E}(V, \tau, \beta)
\end{array}
$$

commutes. So finally, we have

$$
\begin{aligned}
\bar{M}(\Phi)^{*} J(V, \tau, \beta) & =\left[\bar{M}(V, \sigma, \beta), \bar{M}(\Phi)^{*} E^{\bullet}(V, \tau, \beta)\right] \\
& =\left[\bar{M}(V, \sigma, \beta), E^{\bullet}(V, \sigma, \beta)\right] \\
& =J(V, \sigma, \beta),
\end{aligned}
$$

which is Axiom IV.

\section{AxIOM V. Isogenies}

Before we start with the proof, some general remarks. Let $\Phi: \tau \rightarrow \sigma$ be an elementary contraction of stable modular graphs, contracting the edge $\{f, \bar{f}\}$ of $\tau$. Let $a: \tau \rightarrow \tau^{\prime}$ and $b: \sigma \rightarrow \sigma^{\prime}$ be combinatorial morphisms of modular graphs identifying $\tau$ and $\sigma$ as the stabilizations of $\tau^{\prime}$ and $\sigma^{\prime}$, respectively. Finally, let $\Phi^{\prime}: \tau^{\prime} \rightarrow \sigma^{\prime}$ be as follows. We require $\{a(f), a(\bar{f})\}$ to be an edge of $\tau^{\prime}$ and $\Phi^{\prime}: \tau^{\prime} \rightarrow \sigma^{\prime}$ to be the elementary contraction contracting the edge 
$\{a(f), a(\bar{f})\}$. Moreover, we require $\Phi$ to be the stabilization of $\Phi^{\prime}$. To fix notation, denote the vertex onto which $\Phi^{\prime}$ contracts the edge $\{a(f), a(\bar{f})\}$ by $v_{0} \in V_{\sigma^{\prime}}$ and let $v_{1}=\partial_{\tau^{\prime}}(a(f))$ and $v_{2}=\partial_{\tau^{\prime}}(a(\bar{f}))$.

In this situation we get a commutative diagram of algebraic stacks

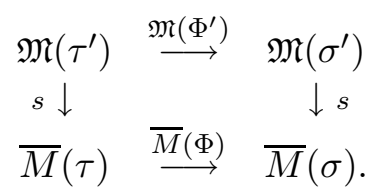

Define $\mathfrak{P}$ to be the fibered product

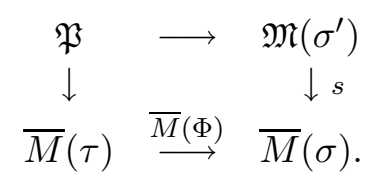

Consider the induced morphism $l: \mathfrak{M}\left(\tau^{\prime}\right) \rightarrow \mathfrak{P}$.

Proposition 8 We have $l_{*}\left[\mathfrak{M}\left(\Phi^{\prime}\right)\right]=s^{*}[\bar{M}(\Phi)]$.

Proof. First note that $\mathfrak{M}\left(\tau^{\prime}\right)$ is irreducible, since $\mathfrak{M}\left(\tau^{\prime}\right)$ is a product of stacks of the form $\mathfrak{M}_{g, n}$, which are irreducible since the stacks $\bar{M}_{g, n}$ are. Moreover, $\mathfrak{M}\left(\tau^{\prime}\right) \rightarrow \mathfrak{P}$ is surjective, so that $\mathfrak{P}$ is irreducible, too.

Secondly, let us remark that there exist non-empty (hence dense) open substacks $\mathfrak{M}\left(\tau^{\prime}\right)^{0} \subset \mathfrak{M}\left(\tau^{\prime}\right)$ and $\mathfrak{P}^{0} \subset \mathfrak{P}$ such that $l$ induces an isomorphism $l^{0}: \mathfrak{M}\left(\tau^{\prime}\right)^{0} \stackrel{\sim}{\rightarrow} \mathfrak{P}^{0}$. In fact, let $\mathfrak{M}\left(\tau^{\prime}\right)^{0}$ be the open substack of $\mathfrak{M}\left(\tau^{\prime}\right)$ characterized by the requirement that the marked curves $C_{v_{1}}$ and $C_{v_{2}}$ be stable. To construct $\mathfrak{P}^{0}$, let $\mathfrak{M}\left(\sigma^{\prime}\right)^{0}$ be the open substack of $\mathfrak{M}\left(\sigma^{\prime}\right)$ where the marked curve $C_{v_{0}}$ is stable. Then set

$$
\mathfrak{P}^{0}=\bar{M}(\tau) \times \bar{M}(\sigma) \mathfrak{M}\left(\sigma^{\prime}\right)^{0} .
$$

These facts imply the claim.

Now let $(\Phi, m): \tau \rightarrow \sigma$ be an elementary isogeny of type forgetting a tail. Let $f \in F_{\tau}$ be the forgotten tail. Let $a: \tau \rightarrow \tau^{\prime}$ and $b: \sigma \rightarrow \sigma^{\prime}$ be as above. Finally, let $\Phi^{\prime}: \tau^{\prime} \rightarrow \sigma^{\prime}$ be the 'adjoint' of a combinatorial morphism of graphs, such that there exists a tail map $m^{\prime}$, a semigroup $A$ and $A$-structures on $\tau^{\prime}$ and $\sigma^{\prime}$ making $\left(\Phi^{\prime}, m^{\prime}\right)$ the elementary isogeny of stable $A$-graphs forgetting the tail $a(f)$. Moreover, we require $\Phi$ to be the stabilization of $\Phi^{\prime}$. 
Let $\mathfrak{P}$ be the fibered product

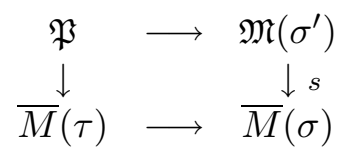

and $\mathcal{C}$ the universal curve over $\mathfrak{M}\left(\sigma^{\prime}\right)$ corresponding to $w \in V_{\sigma^{\prime}}$, where $w$ is the vertex of the forgotten tail. (If $w$ does not exist, i.e. if $\Phi^{\prime}$ is complete, then $\left.\mathcal{C}=\mathfrak{M}\left(\sigma^{\prime}\right).\right)$ As in the proof of Axiom IV we have a morphism $\mathcal{C} \rightarrow \mathfrak{M}\left(\tau^{\prime}\right)$ giving rise to a commutative diagram

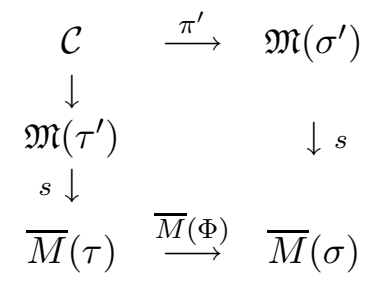

and hence to a morphism $l: \mathcal{C} \rightarrow \mathfrak{P}$.

Proposition 9 We have $l_{*}\left[\pi^{\prime}\right]=s^{*}[\bar{M}(\Phi)]$.

Proof. Again, $\mathcal{C}$ and $\mathfrak{P}$ are irreducible and $l$ induces an isomorphism $l^{0}: \mathcal{C}^{0} \rightarrow \mathfrak{P}^{0}$, where $\mathcal{C}^{0}$ is the restriction of $\mathcal{C}$ to $\mathfrak{M}\left(\sigma^{\prime}\right)^{0}$ and $\mathfrak{P}^{0}=\bar{M} \times \bar{M}(\sigma)$ $\mathfrak{M}\left(\sigma^{\prime}\right)^{0}$. Here $\mathfrak{M}\left(\sigma^{\prime}\right)^{0} \subset \mathfrak{M}\left(\sigma^{\prime}\right)$ is the open substack where $C_{w}$ is stable.

Now let us prove Axiom V. According to [2], Remark 7.2, it suffices to do this for the case that $\Phi: \tau \rightarrow \sigma$ is an elementary isogeny, \#J=1 and $\left(a_{i}, \tau_{i}, \Phi_{i}\right)_{i \in I}$ a pullback. So we shall use notation as in the Definition of pullback ([2], Definition 6.10). We shall include the $H_{2}(V)^{+}$-structures on $\sigma^{\prime}$ and $\tau_{i}(i \in I)$ in the notation. They shall be denoted by $\beta^{\prime}$ and $\beta_{i}(i \in I)$, respectively. The underlying graph of $\left(\tau_{i}, \beta_{i}\right)$ is the same for all $i \in I$. Let us call it simply $\tau^{\prime}$.

Let us first consider the case where $\Phi$ is a contraction.

Lemma 10 We have a cartesian diagram

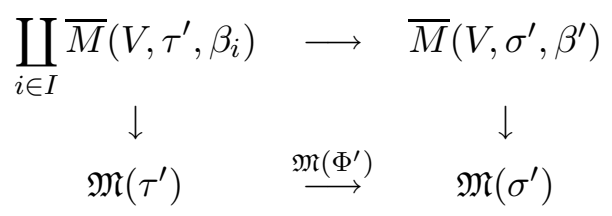


of algebraic $k$-stacks. Moreover,

$$
\mathfrak{M}\left(\Phi^{\prime}\right)^{!} J\left(V, \sigma^{\prime}, \beta^{\prime}\right)=\sum_{i \in I} J\left(V, \tau^{\prime}, \beta^{\prime}\right) .
$$

Proof. The first fact follows immediately from the definitions. The second fact is [1] Proposition 7.2.

Axiom $\mathrm{V}$ will follow by putting Lemma 10 and Proposition 8 together as follows. By Lemma 10 all squares in the following diagram are cartesian.

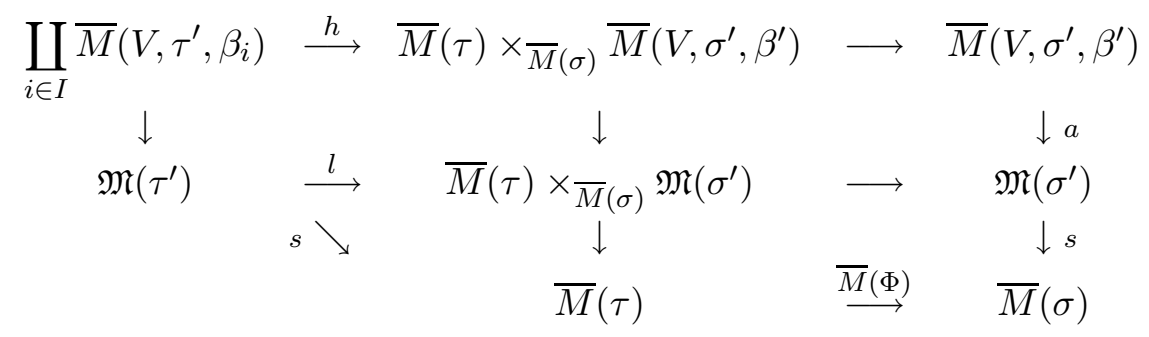

So we may calculate as follows.

$$
\begin{aligned}
\bar{M}(\Phi)^{!} J\left(V, \sigma^{\prime}, \beta^{\prime}\right) & =a^{*} s^{*}[\bar{M}(\Phi)] \cdot J\left(V, \sigma^{\prime}, \beta^{\prime}\right) \\
& =a^{*} l_{*}\left[\mathfrak{M}\left(\Phi^{\prime}\right)\right] \cdot J\left(V, \sigma^{\prime}, \beta^{\prime}\right)
\end{aligned}
$$

(by Proposition 8)

$$
\begin{aligned}
& =h_{*} \mathfrak{M}\left(\Phi^{\prime}\right)^{!} J\left(V, \sigma^{\prime}, \beta^{\prime}\right) \\
& =h_{*} \sum_{i \in I} J\left(V, \tau^{\prime}, \beta_{i}\right)
\end{aligned}
$$

by Lemma 10. This is the context of Axiom V.

The case that $\Phi$ is of type forgetting a tail is similar. Instead of Lemma 10 one uses Axiom IV, and Proposition 8 is replaced by Proposition 9 .

This finishes the proof of Axiom $\mathrm{V}$ and hence the proof of Theorem 6 .

\section{Homogeneous Spaces}

In the case where $V$ is a generalized flag variety, we can give a more explicit construction of Gromov-Witten invariants as follows.

\section{Curves and Principal Bundles}

For a smooth algebraic $k$-group $G$ with Lie algebra $\mathfrak{g}$, we denote by

$$
\mathfrak{H}^{1}(\tau, G)
$$


the $k$-stack of $G$-torsors on $\tau$-marked prestable curves. More precisely, for a $k$-scheme $T$, the category $\mathfrak{H}^{1}(\tau, G)(T)$ is the category of pairs $(C, E)$, where $C=\left(C_{v}\right)_{v \in V_{\tau}}$ is a $\tau$-marked prestable curve over $T$, giving rise to a morphism $f: T \rightarrow \mathfrak{M}(\tau)$, and $E$ is a $G$-torsor on $f^{*} \mathcal{C}(\tau)$.

Let $(C, E)$ be such a pair. Denote by $E_{v}$, for $v \in V_{\tau}$, the $G$-bundle induced by $E$ on $C_{v}$. We call

$$
\operatorname{deg}_{v}(E)=\operatorname{deg}\left(E_{v}\right)=\operatorname{deg}\left(E_{v} \times_{G, A d} \mathfrak{g}\right)
$$

the degree of $E$ at the vertex $v \in V_{\tau}$. The degree thus defines a $\mathbb{Z}_{\geq 0^{-}}$ structure on $\tau$, which is locally constant on $T$. (See [2], Definition 1.6, for $\mathbb{Z}_{\geq 0}$-structures.)

In this way, we get for every $\mathbb{Z}_{>0}$-structure $\alpha$ on $\tau$ an open and closed substack $\mathfrak{H}_{\alpha}^{1}(\tau, G) \subset \mathfrak{H}^{1}(\tau, G)$, the substack of $G$-torsors of degree $\alpha$.

Proposition 11 For every $\mathbb{Z}_{\geq 0}$-structure $\alpha$ on $\tau$ the stack $\mathfrak{H}_{\alpha}^{1}(\tau, G)$ is an algebraic $k$-stack. The canonical morphism

$$
\mathfrak{H}_{\alpha}^{1}(\tau, G) \longrightarrow \mathfrak{M}(\tau)
$$

is smooth of relative dimension

$$
-\chi(\tau) \operatorname{dim} G-\alpha(\tau)
$$

where $\alpha(\tau)=\sum_{v \in V_{\tau}} \alpha(v)$.

Proof. To prove that $\mathfrak{H}^{1}(\tau, G)$ is algebraic, choose a suitable embedding $G \hookrightarrow G L_{n}$ to reduce the case of $G$-bundles to the case of vector bundles, for which it is well-known. The smoothness of $\mathfrak{H}^{1}(\tau, G)$ follows from the fact that $H^{2}\left(C, E \times_{G, A d} \mathfrak{g}\right)=0$ for any $G$-torsor $E$ on a $\tau$-marked prestable curve $C$. The dimension of $\mathfrak{H}^{1}(\tau, G)$ is equal to

$$
\begin{aligned}
-\chi\left(E \times_{G, A d} \mathfrak{g}\right) & =-\operatorname{deg}\left(E \times_{G, A d} \mathfrak{g}\right)-\chi\left(\mathcal{O}_{C}\right) \operatorname{rk}\left(E \times_{G, A d} \mathfrak{g}\right) \\
& =-\alpha(\tau)-\chi(\tau) \operatorname{dim} G
\end{aligned}
$$

by Riemann-Roch.

Maps to $G / P$

Now let $G$ be a reductive algebraic group over $k$ and $P$ a parabolic subgroup of $G$. Then $G / P$ is a smooth projective variety over $k$. Let us assume for 
simplicity that $G$ is split over $k$. The morphism $G \rightarrow G / P$ is a principal $P$-bundle, which we shall denote by $F$.

Let $U_{1}, \ldots, U_{r}$ be the elementary representations of $P$ over $k, V_{1}, \ldots, V_{r}$ the corresponding vector bundles on $G / P$ and $L_{1}, \ldots, L_{r}$ their determinants. For every $i=1, \ldots, r$ we have

$$
V_{i}=F \times_{P} U_{i}
$$

Note that $\operatorname{Pic}(G / P) \otimes \mathbb{Q}$ is spanned by $L_{1}, \ldots, L_{r}$ and that $L_{1}^{-1} \otimes \ldots \times L_{r}^{-1}$ is ample.

Let $H_{2}(G / P)^{+}$be the set of homomorphisms of abelian groups $\psi$ : $\operatorname{Pic}(G / P) \rightarrow \mathbb{Z}$, which are non-negative on ample line bundles. Then we get a canonical injection

$$
\begin{aligned}
H_{2}(G / P)^{+} & \longrightarrow\left(\mathbb{Z}_{\geq 0}\right)^{r} \\
\psi & \longmapsto\left(\psi\left(L_{1}^{-1}\right), \ldots, \psi\left(L_{r}^{-1}\right)\right) .
\end{aligned}
$$

Using this injection we shall think of classes in $H_{2}(G / P)^{+}$as $r$-tuples of non-negative integers.

Let $\mathfrak{g}$ and $\mathfrak{p}$ be the Lie algebras of $G$ and $P$, respectively. We will consider these only as adjoint representations, ignoring the Lie algebra structure. Denote by $\mathfrak{p}$ also the induced vector bundle

$$
F \times{ }_{P, A d \mathfrak{p}}
$$

on $G / P$. Evaluating on the inverse of its determinant defines a morphism

$$
\begin{aligned}
\operatorname{deg}: H_{2}(G / P)^{+} & \longrightarrow \mathbb{Z}_{\geq 0} \\
\psi & \longmapsto \psi\left(\operatorname{det}(\mathfrak{p})^{-1}\right) .
\end{aligned}
$$

This morphism has the property that $\operatorname{deg}(\psi)=0$ implies $\psi=0$.

Remark We have $\operatorname{det} \mathfrak{p} \cong \omega_{G / P}$. In particular, $\operatorname{deg} \psi=-\psi\left(\omega_{G / P}\right)$.

Now fix an $H_{2}(G / P)^{+}$-graph $(\tau, \beta)$, with underlying modular graph $\tau$. Let $(\widetilde{\tau}, \widetilde{\beta})$ be the $\mathrm{H}_{2}(G / P)^{+}$-graph obtained by contracting all edges of $\tau$.

Consider the algebraic $k$-stacks $\mathfrak{H}^{1}(\tau, G)$ and $\mathfrak{H}^{1}(\tau, P)$. Since $G$ is reductive, any $G$-torsor on a curve has degree zero, and thus

$$
\mathfrak{H}^{1}(\tau, G) \longrightarrow \mathfrak{M}(\tau)
$$


is smooth of relative dimension

$$
-\chi(\tau) \operatorname{dim} G
$$

If $E$ is a $P$-torsor, then associated to $U_{1}, \ldots, U_{r}$ we have associated vector bundles $E_{i}=E \times{ }_{P} U_{i}$, for $i=1, \ldots, r$, and thus we may associate to $E$ the multi-degree

$$
\operatorname{mult}-\operatorname{deg}(E)=\left(-\operatorname{deg}\left(E_{1}\right), \ldots,-\operatorname{deg}\left(E_{r}\right)\right) .
$$

Let $\mathfrak{H}_{\beta}^{1}(\tau, P)$ be the open and closed substack of $\mathfrak{H}^{1}(\tau, P)$ of $P$-torsors whose multi-degree is equal to $\beta$.

Let $\alpha=\operatorname{deg} \beta$ be the $\mathbb{Z}_{\geq 0}$-structure on $\tau$ associated to $\beta$. Then we have

$$
\mathfrak{H}_{\beta}^{1}(\tau, P) \subset \mathfrak{H}_{-\alpha}^{1}(\tau, P),
$$

so that by Proposition 11 the stack $\mathfrak{H}_{\beta}^{1}(\tau, P)$ is smooth of relative dimension

$$
-\chi(\tau) \operatorname{dim} P-\beta(\tau)\left(\omega_{G / P}\right)
$$

over $\mathfrak{M}(\tau)$.

Now let $\mathfrak{M}(G / P, \tau, \beta)$ be the stack of maps from $\tau$-marked prestable curves to $G / P$ of class $\beta$. More precisely, for a $k$-scheme $T$, the objects of $\mathfrak{M}(G / P, \tau, \beta)(T)$ are triples $(C, x, f)$, where $(C, x)$ is a $\tau$-marked prestable curve over $T$ and $f=\left(f_{v}\right)_{v \in V_{\tau}}$ is a family of $k$-morphisms $f_{v}: C_{v} \rightarrow G / P$ such that

1. for all $i \in F_{\tau}$ we have $f_{\partial(i)}\left(x_{i}\right)=f_{\partial\left(j_{\tau}(i)\right)}\left(x_{j_{\tau}(i)}\right)$,

2. for all $v \in V_{\tau}$ we have $f_{v_{*}}\left[C_{v}\right]=\beta(v)$.

Remark If $(\tau, \beta)$ is stable, then $\bar{M}(G / P, \tau, \beta)$ is an open substack of $\mathfrak{M}(G / P, \tau, \beta)$.

Note that $G_{\tau}^{V_{\tau}}$ acts on $\mathfrak{M}(G / P, \tau, \beta)$ as follows. An element $\left(g_{w}\right)_{w \in V_{\tau}}$ of $G^{V_{\tau}}$ takes $\left(C, x,\left(f_{v}\right)_{v \in V_{\tau}}\right)$ to $\left(C, x,\left(g_{\phi(v)} \circ f_{v}\right)_{v \in V_{\tau}}\right)$, where $\phi: \tau \rightarrow \widetilde{\tau}$ is the structure contraction. Let

$$
\mathfrak{M}(G / P, \tau, \beta) / G^{V_{\tau}}
$$

be the stack-theoretic quotient of this action. This is an abuse of notation, since this is a left and not a right action. 
We shall let $G^{V_{\tau}}$ act trivially on $\mathfrak{M}(\tau)$ and denote by

$$
\mathfrak{M}(\tau) / G^{V_{\tau}}
$$

the quotient.

Proposition 12 There is a natural cartesian diagram of algebraic $k$-stacks

$$
\begin{array}{ccc}
\mathfrak{M}(G / P, \tau, \beta) / G^{V \tau} & \stackrel{\kappa}{\longrightarrow} & \mathfrak{H}_{\beta}^{1}(\tau, P) \\
\eta \downarrow & & \downarrow \\
\mathfrak{M}(\tau) / G^{V} & \stackrel{\iota}{\longrightarrow} & \mathfrak{H}^{1}(\tau, G) .
\end{array}
$$

The vertical maps are representable, the horizontal maps are local immersions.

Proof. This is essentially the fact that a map to $G / P$ is the same as a principal $P$-bundle with a trivialization of the associated $G$-bundle.

The morphism $\iota$ is a local regular immersion with normal bundle $R^{1} \pi_{*} \mathcal{O} \otimes \mathfrak{g}$. Thus the normal cone $C(\tau, \beta)$ of $\mathfrak{M}(G / P, \tau, \beta) / G_{\tau}^{V_{\tau}}$ in $\mathfrak{H}_{\beta}^{1}(\tau, P)$ is a cone in

$$
\mathfrak{n}(\tau, \beta)=\eta^{*} R^{1} \pi_{*} \mathcal{O} \otimes \mathfrak{g}
$$

Pulling back to $\mathfrak{M}(G / P, \tau, \beta)$ and, if $(\tau, \beta)$ is stable, to $\bar{M}(G / P, \tau, \beta)$ defines $G_{\tau}^{V \tau}$-equivariant cones, which we shall still denote $C(\tau, \beta)$, inside equivariant vector bundles, which we shall still denote by $\mathfrak{n}(\tau, \beta)$.

Let us now assume that $(\tau, \beta)$ is stable. Then we may intersect the cone $C(\tau, \beta)$ over $\bar{M}(G / P, \tau, \beta)$ with the zero section of the vector bundle $\mathfrak{n}(\tau, \beta)$, to define a cycle class

$$
J(\tau, \beta) \in A_{\operatorname{dim}(G / P, \tau, \beta)}(\bar{M}(G / P, \tau, \beta))
$$

with rational coefficients. Note that $C(\tau, \beta)$ is pure of the correct dimension, since it is constructed as a normal cone inside a smooth stack of the correct dimension.

Proposition 13 The collection of cycle classes $J(\tau, \beta)$ is the orientation of $\bar{M}$ over $\mathfrak{G}_{s}(G / P)$ defined using the intrinsic normal cone.

Proof. This follows from 迎 Example 7.6, since

$$
\left(R \pi_{*} f^{*} T_{G / P}\right)^{\vee}=\kappa^{*} L_{\mathfrak{H}_{\beta}^{1}(\tau, P) / \mathfrak{H}^{1}(\tau, G)}^{\bullet}
$$

Remark As a corollary we get that the orientation classes $J(\tau, \beta)$ are $G^{V_{\tau}}$ invariant. The same is then true for the Gromov-Witten invariants. 


\section{References}

[1] K. Behrend and B. Fantechi. The intrinsic normal cone. Preprint, 1996.

[2] K. Behrend and Yu. Manin. Stacks of stable maps and Gromov-Witten invariants. To appear in Duke Mathematical Journal.

[3] A. Grothendieck. Techniques de construction et théorèmes d'existence en géométrie algébrique IV: Les schémas de Hilbert. Séminaire Bourbaki, 13e année(221), 1960-61.

[4] F. Knudsen. The projectivity of the moduli space of stable curves, II: The stacks $M_{g, n}$. Math. Scand., 52:161-199, 1983.

[5] M. Kontsevich and Yu. Manin. Gromov-Witten classes, quantum cohomology, and enumerative geometry. Commuications in Mathematical Physics, 164:525-562, 1994.

behrend@math.ubc.ca 